Provided for non-commercial research and education use. Not for reproduction, distribution or commercial use.

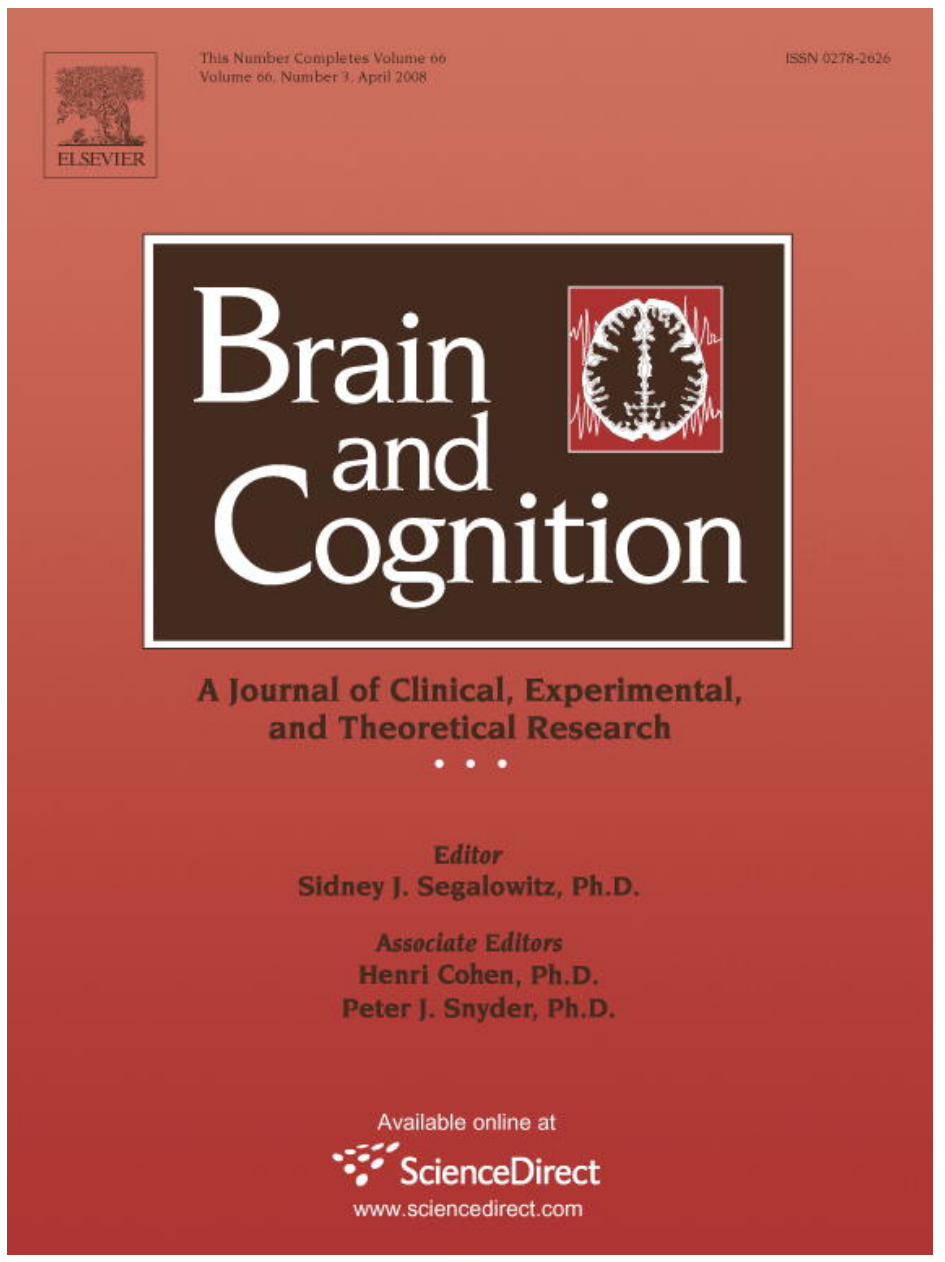

This article was published in an Elsevier journal. The attached copy

is furnished to the author for non-commercial research and education use, including for instruction at the author's institution, sharing with colleagues and providing to institution administration.

Other uses, including reproduction and distribution, or selling or licensing copies, or posting to personal, institutional or third party websites are prohibited.

In most cases authors are permitted to post their version of the article (e.g. in Word or Tex form) to their personal website or institutional repository. Authors requiring further information regarding Elsevier's archiving and manuscript policies are encouraged to visit: 


\title{
Memory abilities in Williams syndrome: Dissociation or developmental delay hypothesis?
}

\author{
Adriana Sampaio ${ }^{\mathrm{a}, \mathrm{b}, *}$, Nuno Sousa ${ }^{\mathrm{b}}$, Montse Férnandez ${ }^{\mathrm{a}}$, \\ Margarida Henriques ${ }^{c}$, Óscar F. Gonçalves ${ }^{a}$ \\ a Department of Psychology, University of Minho, Campus de Gualtar, 4710-057 Braga, Portugal \\ ${ }^{\mathrm{b}}$ Life and Health Sciences Research Institute, University of Minho, Braga, Portugal \\ ${ }^{\mathrm{c}}$ Faculty of Psychology and Education Sciences, University of Porto, Porto, Portugal
}

Accepted 14 September 2007

Available online 24 October 2007

\begin{abstract}
Williams syndrome (WS) is a neurodevelopmental genetic disorder often described as being characterized by a dissociative cognitive architecture, in which profound impairments of visuo-spatial cognition contrast with relative preservation of linguistic, face recognition and auditory short-memory abilities. This asymmetric and dissociative cognition has been also proposed to characterize WS memory ability, with sparing of auditory short-term memory and impairment of spatial and long-term memory abilities. In this study, we explored the possibility of a double memory dissociation in WS (short- versus long-term memory; verbal versus visual memory). Thus, verbal memory abilities were assessed using California Verbal Learning Test and Digit Span and Rey-Osterrieth Complex Figure and Corsi Blocks was used to assess visual-spatial memory abilities. Overall, WS subjects were found to present a generalized significant impairment in verbal and visuo-spatial components either in short- or long-term memory. In sum, data from this study brings support for a developmental delay hypothesis, rather than a double dissociation within memory systems in WS.
\end{abstract}

(c) 2007 Elsevier Inc. All rights reserved.

Keywords: Williams syndrome; Memory dissociation; Neurodevelopment

\section{Introduction}

Williams syndrome (WS) is a neurodevelopmental disorder, with a prevalence of 1 in 7500 (Stromme, Bjornstad, \& Ramstad, 2002), characterized by a deletion on chromosome 7 q11.22-23 (Korenberg et al., 2000). Of further note, WS patients have an unusual phenotype, which includes a distinctive profile of physical, medical, neurocognitive and neuroanatomical characteristics. Physical characteristics include craniofacial and cardiac/pulmonary abnormalities, growth delay, hypercalcemia, hyperacusis and feeding difficulties (Metcalfe, 1999). The other main component of the WS phenotype is a neurodevelopment/cognitive profile

\footnotetext{
* Corresponding author. Address: Department of Psychology, University of Minho, Campus de Gualtar, 4710-057 Braga, Portugal.

E-mail address: adriana.sampaio@iep.uminho.pt (A. Sampaio).
}

characterized by mental retardation and an asymmetrical development, with weak and strong areas of performance. Specifically, severe impairment of visuo-spatial cognition has been described as coexisting with a relative preservation of face recognition, auditory short-term memory, language and narrative skills (Bellugi, Lichtenberger, Jones, Lai, \& St. George, 2000; Gonçalves et al., 2004, 2005; Mervis et al., 2000).

This uneven cognitive profile of relative strengths and weaknesses also seems to be evident within each cognitive domain. For example, several studies found evidence for the existence of a good short-term verbal memory, even when compared with normal development controls (Bellugi, Wang, \& Jernigan, 1994; Mervis, Morris, Bertrand, \& Robinson, 1999; Nichols et al., 2004). However, several authors have been finding that this short-term verbal memory strength is not generalized across different memory sys- 
tems (Vicari, Bellucci, \& Carlesimo, 2003; Vicari, Brizzolara, Carlesimo, Pezzini, \& Volterra, 1996). For example, differences of ability in short- and long-term memory storage systems were found in a study by Vicari et al. (1996). The authors compared memory performance on Digit Span, Corsi Blocks, Rey-Osterrieth Complex Figure and a word list task in a WS group and a verbal mental age matched group. While a spatial span (Corsi Blocks) was found to be significantly reduced in the WS group, no significant differences were found in terms of verbal span. Additionally, no primacy effect in the serial position curve was found with the WS group. The authors interpreted their results as an evidence for the impairment of semantic long-term memory along with the preservation of shortterm phonological memory. This claim was further supported by Nichols et al. (2004) using California Verbal Learning Test (CVLT).

More recently, Brock, Brown, and Boucher (2006) replicated the Vicari et al. (1996) study, introducing some procedural changes. Namely, the serial position curve was obtained in three different presentation conditions: different words, repeated words in different order and a repeated list. Using this strategy, both groups (WS and control) showed a similar pattern of recency, but no primacy effects in serial position curve. Additionally, participants were trained with a cumulative rehearsal strategy in order to determine the primacy effect extension. Again, no significant differences were found between groups in global performance (i.e., the rehearsal strategy was not associated with a better performance), but now, a primacy effect was observable in both groups. The authors suggested that there is not a selective long-term memory deficit in WS and that the absence of a primacy effect in WS may related with the inability in using rehearsal strategies.

A dissociation in working memory components (between phonological loop and visuo-spatial sketchpad) has been also proposed for characterizing cognitive profile of WS (Jarrold, Baddeley, \& Hewes, 1999). These authors tested the hypothesis of the preservation of phonological loop coexisting with impaired visuo-spatial sketchpad in WS. Twenty-five children with Down syndrome (DS) were compared with 16 children and young adults with WS and 17 children with moderate learning disabilities, in short-term verbal memory (Digit Span) and short-term visuo-spatial memory (Corsi Blocks). The results revealed that WS had the lowest score in short-term visuo-spatial memory contrasting with a superior Digit Span performance. In a second study, the authors matched three groups in terms of nonverbal mental age (WS, learning disabilities and a normal development), comparing their performance in three short-term memory measures: one verbal (Digit Span) and two nonverbal (Corsi Span and Pattern Memory). Again, the results showed that WS group had significant lower scores in Corsi Blocks. However, in the Pattern Memory test, WS group was not impaired with respect to the control group. The authors concluded suggesting the existence of a specific impairment in visuo-spatial sketchpad and a relative sparing of the phono- logical loop in WS. However, more recently, Jarrold, Baddeley, and Phillips (2007) proposed that these visuo-spatial memory deficits may be secondary to visuo-spatial processing difficulties, a main feature of WS cognitive profile (Mervis et al., 2000). Indeed, WS patients exhibit difficulties in several aspects of visual-spatial cognition, including spatial representations (Farran \& Jarrold, 2005; Hoffman, Landau, \& Pagani, 2003), abilities that are necessary to perform some visual-spatial memory tasks. The preservation of WS performance in Pattern Memory Test was interpreted by Vicari and colleagues (2003) as suggesting that WS represents a natural case of dissociation within the two components of visuo-spatial sketchpad, in which visual perception is relatively preserved. These results were further investigated with respect to long-term memory system, showing that individuals with WS had impairments in learning visual-spatial material, although a typical performance in visual-object long-term memory (Vicari, Bellucci, \& Carlesimo, 2005).

Overall, previous studies have been showing evidence for an uneven performance in WS individuals across different memory tests. The current study attempts to confirm the existence in WS of a double dissociation in memory systems between short- versus long-term memory as well as between verbal and visuo-spatial working memory components.

\section{Method}

\subsection{Participants}

A group of 14 individuals with WS ( 7 male and 7 female participants), with age range between 8 and 29 years $(M=16.79, \quad S D=5.68$; mean Full Scale IQ $=49.14$, $S D=7.50$, range 40-61) was compared with a normal development group ( $N=14,5$ male and 9 female) ranging in age from 8 to 29 years $(M=17.93, S D=6.10$; mean Full Scale IQ $=110.50, S D=10.48$, range 90-124). Given that the neurological profile of WS may change across development (Gagliardi, Martelli, Burt, \& Borgatti, 2007), two subgroups were compared on the basis of chronological age for both WS and normal development participants: children $(N=5$, age $8-14$ years) and adult $(N=9$, age 15-29 years) subgroups.

WS participants were recruited at Genetic Medical Institute (Portugal) and Genomic Foundation in Galicia (Spain). WS diagnoses were made by FISH confirmation of elastin gene deletion (Korenberg et al., 2000). Controls were typically developing individuals without evidence of psychiatric, neurological disorder or cognitive impairment. Each participant gave written informed consent for their participation in the study via consent forms, after a complete description of the study.

\subsection{Instruments}

To assess general cognitive functioning (Full Scale IQ), participants 8-16 years of age were administered the 
Wechsler Intelligence Scale for Children-Third Edition (WISC-III) (Wechsler, 1991), while subjects over 16 years old were administered the Wechsler Adult Intelligence Scale-Third Edition (WAIS-III) (Wechsler, 1997).

California Verbal Learning Test (CVLT) - children (Delis, Kramer, Kaplan, \& Ober, 1994) and adult (Delis, Kramer, Kaplan, \& Ober, 2000) version-clinical instrument that allows a complete description of memory and learning abilities. For this study both children and adult versions were used. This test consists of two shopping lists (A and B) that are read to the participant. List A is presented five times and list $\mathrm{B}$ is presented after the fifth trial of $\mathrm{A}$ list. In list $\mathrm{A}$, the following measures were analyzed: number of words recalled in the first and fifth trials and total number of correct words for all five trials. For list $\mathrm{B}$, the total number of recalled items was also reported. Short- and long-term delay, recognition performance and discriminability responses were calculated, as well as false positives and intrusions. To analyze the primacy and recency effect, a Position Serial Curve was built for both groups. This was calculated computing the mean number of recalled words in each position that is repeated five times. Given the fact that the child version is composed by 15 words and adults version by 16 words, we added one position in the intermediate region (between position 7 and 8 - that was the mean of recalled words in these positions). The option for this intermediate region is due to the fact that, excluding primacy and recency effects, the probability of recalling the remaining items is practically equivalent and produce the flat component of serial position curve. This instrument has a wide field of application, specifically in children with learning abilities, including Specific Language Impairment, Down syndrome, WS and other etiologies (Bellugi et al., 1994; Nichols et al., 2004).

Digit Span - the Digit Span subtest from adult and children version of the Wechsler Intelligence Scale (Wechsler, 1991, 1997) was used to evaluate verbal memory immediate span. Forward and backward digit span were analyzed separately, since the performance in each task is associated with different cognitive abilities. Backward digit span is related to working memory ability, specifically central executive, and forward digit span is related to phonological loop. In this sense, this subtest allows the evaluation of two components of Baddeley's and Hitch (Baddeley \& Hitch, 1974) working memory model - the phonological loop and central executive.

Corsi Blocks (Corsi, 1972) — this test consists in a series of nine blocks arranged irregularly, that are tapped by the examiner in different sequences of increasing length ( 2 trials for sequence). After each tapped sequence, the subject must reproduce it, until fails both trials of a sequence (in forward and backward order). We used Spatial Blocks as a measure visuo-spatial sketchpad of Baddeley and Hitch (1974) working memory model.

Rey-Osterrieth Complex Figure (Rey, 1959) — this test consists in reproducing a geometric figure with no concrete meaning. The drawings were coded with a developmental coding system (Bernstein \& Waber, 1996). Briefly, this system has three scores (Organization, Accuracy and Errors) and a rating (style) for copy and recall condition. The organization score is based on criteria features of the complex figure (alignments and intersections). The style is rated in one of three categories: part-oriented, intermediate and configurational. Accuracy scores account for the number of line segments (Structural Elements Accuracy) and internal details (Incidental Elements Accuracy). Although not reported in this study, the number of errors can also be object of analysis.

\subsection{Procedure}

After collecting data about sociodemographic characteristics, diagnosis, clinical history and general cognitive functioning, all participants were submitted to four memory tasks: two verbal memory and two visuo-spatial memory tasks. For Digit Span and Corsi Blocks, we calculated the mean immediate memory span (IMS) for each participant. Each span task started with a two items (digits or spatial position) that increased sequentially with two trials in each length. Test stopped when both lists at one length were recalled incorrectly. IMS was defined as the total of not related items that a person is able to reproduce, in the same order, following one presentation. IMS measure was calculated by the following equation: $(A+B) / C,(A$, referring to the longest length in which the participant has correct answers in both trials; $B$, the remaining correct answers; $C$, number of trials in each length-2).

To analyze relative levels of impairments across different tests (verbal and visual) raw scores were converted into $z$ scores with respect to normal development group. Thus, Digit and Corsi Span (measures of phonological loop and visuo-spatial sketchpad) were standardized into $z$ scores. Also, long-term free recall in California Verbal Learning Test and a memory index in Rey-Osterrieth Complex Figure (sum of structural and incidental elements in memory condition), two measures of verbal and visual-spatial long-term memory, were converted in $z$ scores.

\subsection{Data analysis}

Descriptive analyses were used to characterize the group of participants. To determine differences between the WS and control groups with respect to the different memory measures, Student's $t$ test were used, after verifying normality of distribution and variance homogeneity by Shapiro-Wilks test and Levene's test, respectively. When these criteria were not satisfied, Mann-Whitney tests were used.

Considering the increased number of comparisons in CVLT and to limit the chance of falsely reject the null hypothesis, we adjusted the significance of $p$ value to $<$.01. A paired sample $t$ test was used to analyze WS $z$ scores across different memory tests performance. Pearson's correlation was used as an association measure and Chi-square test was used for nominal data (figure approaching strategy in Rey-Osterrieth task). In addition, 
a two-way analysis of variance (ANOVA) was conducted with Diagnose and Age Group as Fixed Factors and the different memory measures were used as dependent variables.

\section{Results}

The results are presented for each test. First, we present the scores obtained in CVLT, Digit Span and Corsi Blocks in the two comparison groups. Finally, Rey-Osterrieth Complex Figure results are reported.

\subsection{Results in CVLT in both groups}

As can be observed observe in Fig. 1 and Table 1, individuals with WS showed lower scores in almost CVLT variables. Two exceptions are worth mention. First, immediate

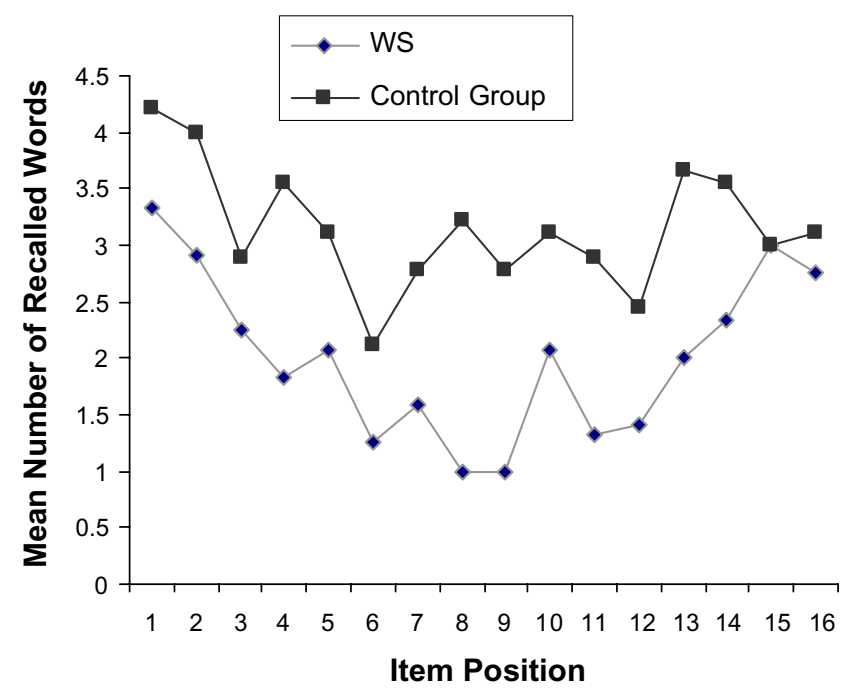

Fig. 1. Serial position curve in WS and control group. memory span to the first trial of A list was not found to be statistically different between WS and control $(Z=-.283$, $p=.777)$. Additionally, although there is an increase in recalled items from trial to trail, a similar learning curve was observed in both groups $(t(26)=-1.578, p=.128$, effect size $=-0.65$ ). Also, when age subgroups were analyzed, there was an Age Group effect $(F(1,24)=9.828$, $p<.01)$ and an interaction between Age Group and Diagnosis $(F(1,24)=3.123, p<.05)$ with younger groups exhibiting a higher learning curve, being more pronounced this difference in WS group. The items span in the last trial was found to be significantly higher in control group $(t(26)=-5.616, p<.001$, effect size $=-2.18)$. This difference is reflected in total A list recalled words $(t(26)=-5.912, p<.001$, effect size $=-2.30)$.

Comparing first trial A and B Lists total recalled items, we found that while normal performance increases in normal controls from list A $(M=5.64, S D=1.08)$ to list $\mathrm{B}$ $(M=6.79, S D=1.63)$ exactly the opposite occurs in WS with a decrease from first trial list A $(M=5.21$, $S D=1.89)$ to list B $(M=4.79, S D=1.42)$. When list B performance was analyzed accordingly to age subgroups, there was a Diagnose effect $(F(1,24)=16.336, p<.001)$ and Age Group effect $(F(1,24)=14.571, p<.01)$ in IMS (list B), with younger subgroups performing better.

With respect to retention interval, WS participants were found to recall significantly less words both, in short delay $(t(26)=-6.000, p<.001$, effect size $=-2.27)$ and long delay interval $(t(26)=-6.408, \quad p<.001, \quad$ effect size $=-2.42$ ). Thus, the use of both semantic strategies that facilitate information recovery are less used by WS group, although no significant differences were found between groups with respect to the use of serial strategies $(t(26)=-2.293, p=.022$, effect size $=-0.87)$. Again, the score in the recognition task is significantly lower in WS group with respect to control group $(Z=-3.398$, $p<.01)$. This performance may be affected by the large

Table 1

Mean CVLT raw scores in WS and control group

\begin{tabular}{|c|c|c|c|c|c|}
\hline \multirow[t]{2}{*}{ CVLT } & \multicolumn{2}{|l|}{$\mathrm{WS}(N=14)$} & \multicolumn{2}{|c|}{ Control group $(N=14)$} & \multirow{2}{*}{$\begin{array}{l}t(26) \\
Z\end{array}$} \\
\hline & $M(S D)$ & Range & $M(S D)$ & Range & \\
\hline IMS ( $1^{\circ}$ trial $\mathrm{A}$ list $)$ & $5.21(1.89)$ & $2-7$ & $5.64(1.08)$ & $4-8$ & $-.283^{\mathrm{a}}$ \\
\hline IMS ( $5^{\circ}$ trial A list) & $8.14(2.60)$ & $2-12$ & $12.71(1.60)$ & $10-15$ & $-5.616^{*}$ \\
\hline IMS (B list) & $4.79(1.42)$ & $3-8$ & $6.79(1.63)$ & $5-10$ & $-3.129^{*}, \mathrm{a}$ \\
\hline Total recalled items in the 5 trials & $32.14(9.51)$ & $15-47$ & $49.64(5.68)$ & $40-60$ & $-5.912^{* *}$ \\
\hline Learning curve & $.84(1.13)$ & $0.46-2.80$ & $1.44(0.70)$ & $0.46-2.50$ & -1.578 \\
\hline Short-term free recall & $6.86(2.01)$ & $4-12$ & $11.43(2.02)$ & $7-15$ & $-6.00^{* *}$ \\
\hline Long-term free recall & $7.29(1.87)$ & $4-11$ & $12.14(2.13)$ & $7-15$ & $-6.408^{* *}$ \\
\hline Semantic strategies & $11.00(5.49)$ & $1-18$ & $25.50(12.38)$ & $11-46$ & $-3.206^{\mathrm{a}, *}$ \\
\hline Serial strategies & $4.21(2.83)$ & $1-11$ & $6.71(2.89)$ & $3-14$ & $-2.293^{\mathrm{a}}$ \\
\hline Intrusions & $10.07(7.40)$ & $2-23$ & $2.79(2.89)$ & $0-8$ & $-3.047^{\mathrm{a}, *}$ \\
\hline Recognition task & $11.14(3.39)$ & $5-15$ & $14.71(1.44)$ & $10-16$ & $-3.398^{\mathrm{a}, *}$ \\
\hline False positives in recognition task & $2.71(2.61)$ & $0-9$ & $0.14(.36)$ & $0-1$ & $-3.036^{\mathrm{a}, *}$ \\
\hline Discriminability & $84.50(8.14)$ & $71.10-97.73$ & $97.57(4.22)$ & $84.10-100$ & $-4.011^{\mathrm{a}, *}$ \\
\hline
\end{tabular}

\footnotetext{
a Mann-Whitney test was used to compare both groups.

${ }^{*} p<.01$.

** $p<.001$.
} 
amount of false positive that WS $(Z=-3.036, p<.01)$ identify. Indeed, one of the major difficulties of WS has to do with their inability to discriminate relevant information shown in by a significant lower discriminability index $(Z=-4.011, p<.01)$ along with a significant increase in the number of intrusions $(Z=-3.047, p<.01)$.

\subsection{Serial position curve}

Although there is a reduced span in recalled items, individuals with WS demonstrate a serial position curve like normal controls, characterized by a U-shape with recency and primacy effect (Fig. 1).

\subsection{Results in Digit Span test}

As can be seen in Table 2 WS participants have significantly lower scores in Digit Span test when compared with the control group as reflected in IMS either in forward $(Z=-3.758, \quad p<.001) \quad$ or backward conditions $(Z=-2.908, p<.01)$. However, when comparing the magnitude difference of recalled items in forward and backward conditions, no differences were found between WS and controls $(Z=-.211, p=.833)$. When age subgroups were considered, a significant effect was found for both Diagnosis $(F(1,24)=17.630, p<.001)$ and Age $(F(1,24)=4.342$, $p<.05$ ) for Digit Span (backward), with the adult subgroups showing an increased immediate memory span for digits.

\subsection{Results in Corsi Blocks}

As can be seen in Table 3, the individuals with WS had a significantly lower score in Corsi Blocks in both forward $(Z=-4.096, p<.001)$ and backward recall $(Z=-4.382$, $p<.001)$. Again, in spite of these difference, when comparing the magnitude difference of recalled spatial positions in forward and backward conditions, no differences were found between WS and controls $(Z=-1.885, p=.059)$.

\subsection{Performance in Digit Span and Corsi Span}

The results presented above show evidence for the existence of significantly lower scores in WS for both verbal and spatial span. However, when comparing both groups in Digit Span and Corsi Block, we found that, although WS have a lower span in both tasks, they exhibit a similar performance pattern in both, forward $(t(26)=-1.402$, $p=.174$, effect size $=0.57$ ) and backward conditions $(t(26)=-1.084, p=.289$, effect size $=-0.44)$. That is, the magnitude of the difference in Digit Span scores and Corsi Blocks scores (in the two recall conditions) is similar in both groups.

A comparison of the degree of impairment in verbal and visuo-spatial short-term memory measures was also analyzed by standardizing the data of the clinical group relative to normal development group. Thus $z$ scores were calculated for Digit and Corsi Span in forward condition and no differences were found between performance in WS $z$ scores in these two tests $(t(13)=1.214, p=.250)$, two measures of the phonological loop and visuo-spatial sketchpad components, respectively.

It is interesting also to note that a significant positive correlation was found between the Full Scale IQ and immediate memory span for both, forward $(r=.750, p<.001)$ and backward Digit Span $(r=.625, p<.01)$ as well as with immediate memory span for spatial positions in forward $(r=.740, \quad p<.001)$ and backward position $(r=.938$, $p<.001)$.

Table 2

Performance in Digit Span and immediate memory span in WS and control group

\begin{tabular}{|c|c|c|c|c|c|}
\hline \multirow[t]{2}{*}{ Digit Span } & \multicolumn{2}{|c|}{$\mathrm{WS}(N=14)$} & \multicolumn{2}{|c|}{ Control group $(N=14)$} & \multirow[t]{2}{*}{$Z$} \\
\hline & $M(S D)$ & Range & $M(S D)$ & Range & \\
\hline Forward & $6.50(.80)$ & $5-8$ & $9.64(1.60)$ & $7-12$ & $-4.316^{* *}$ \\
\hline Backward & $3.00(1.04)$ & $2-5$ & $6.29(2.55)$ & $2-11$ & $-3.599^{*}$ \\
\hline IMS - forward & $4.25(.40)$ & $3.5-5.0$ & $5.68(.89)$ & $4-7$ & $-3.758^{* *}$ \\
\hline IMS — backward & $2.75(.75)$ & $2-4$ & $4.07(1.25)$ & $3-6.5$ & $-2.908^{*}$ \\
\hline
\end{tabular}

${ }^{*} p<.01$.

** $p<.001$.

Table 3

Performance and IMS in Corsi Blocks in WS and control group

\begin{tabular}{|c|c|c|c|c|c|}
\hline \multirow[t]{2}{*}{ Corsi Blocks } & \multicolumn{2}{|c|}{$\mathrm{WS}(N=14)$} & \multicolumn{2}{|c|}{ Control group $(N=14)$} & \multirow[t]{2}{*}{$Z$} \\
\hline & $M(S D)$ & Range & $M(S D)$ & Range & \\
\hline Forward & $4.08(1.08)$ & $3-7$ & $7.71(1.64)$ & $5-10$ & $-4.096^{* *}$ \\
\hline Backward & $2.33(0.78)$ & $1-4$ & $7.29(1.27)$ & $5-9$ & $-4.382^{* *}$ \\
\hline IMS - forward & $3.04(0.54)$ & $2.5-4.5$ & $4.89(0.79)$ & $3.5-6$ & $-4.130^{* *}$ \\
\hline IMS_-backward & $2.13(0.48)$ & $1-3$ & $4.64(0.63)$ & $3.5-5.5$ & $-4.382^{* *}$ \\
\hline
\end{tabular}

** $p<.001$ 
Table 4

Performance in Rey-Osterrieth Complex Figure in WS and control group

\begin{tabular}{|c|c|c|c|c|c|}
\hline \multirow[t]{2}{*}{ Rey-Osterrieth-Memory recall } & \multicolumn{2}{|c|}{$\mathrm{WS}(N=14)$} & \multicolumn{2}{|c|}{ Control group $(N=14)$} & \multirow[t]{2}{*}{$Z$} \\
\hline & Mdn & Range & Mdn & Range & \\
\hline \multirow[t]{2}{*}{ Organization } & 2 & $1-5$ & 3 & $2-13$ & $-3.794^{* *}$ \\
\hline & $M(S D)$ & Range & $M(S D)$ & Range & \\
\hline Structural elements & $11.46(5.47)$ & $0-18$ & $22.36(3.75)$ & $12-25$ & $-4.126^{* *}$ \\
\hline Incidental elements & $9.31(8.15)$ & $0-27$ & $21.43(8.67)$ & $7-35$ & $-3.185^{*}$ \\
\hline
\end{tabular}

${ }^{*} p<.01$
${ }^{* *} p<.001$

\subsection{Results in Rey-Osterrieth Complex Figure-recall trial}

As can be seen in Table 4, individuals with WS show evidence of a significant inferior quality in the figure's organization $(Z=-3.794, p<.001)$. No significant relationship between groups in the figure approaching strategy $\left(\chi^{2}(2)=3.196, p=.202\right)$. Indeed, the configurational strategy was the most frequently used in control $(85.7 \%)$ and WS (64.3\%) participants. Additionally, WS recalled significantly less structural $(Z=-4.126, p<.001)$ and incidental elements $(Z=-3.185, p<.01)$. In WS group, a positive significant correlation between age and structural and incidental elements $(r=.799, p<.01)$.

Given the fact that participants' performance in recall trial may be either related to copy trial performance or to a memory deficit, the memory score (structural and incidental elements) was computed as a ratio of the score obtained in memory and copy trials. No significant differences were found between WS and control group $(Z=-1.335, p=.182)$ suggesting that the visual information loss after a delay was similar in both groups.

Also, WS group $z$ scores on verbal and visual-spatial long-term memory measures were compared, specifically, long-term free recall in California Verbal Learning Test and a memory index in Rey-Osterrieth Complex Figure (sum of structural and incidental elements in memory condition). Again, no differences were found between performance in California Verbal Learning Test and in ReyOsterrieth Complex Figure $(t(13)=-.041, p=.968)$, two measures of verbal and visual-spatial long-term memory, respectively.

\section{Discussion}

Overall, the results of this study demonstrate that WS individuals have a global impairment of working memory components (phonological loop and visuo-spatial sketchpad). In addition, we failed to observe significant differences in short- versus long-term memory storage systems, as they were equally affected in WS subjects.

The differences between our study and some studies, who found evidence for the dissociation hypothesis in WS memory, may be due to different criteria in selecting the control group. Our study used a matching chronological age control group. The comparison group choice is a determinant aspect in developmental neurocognitive studies. For example, there is evidence that children with normal development do not show primacy effect around 6-7 years old (Brock et al., 2006), visual span is unaltered between ages 5 and 7 (increasing significantly between years 7 and 10) and spatial span shows developmental leaps (Vicari et al., 2003). These different developmental trends suggest the involvement of different cognitive processes in verbal, visual and spatial memory tasks that must be considered in control group selection. However, these aspects are not considered in the majority of studies (Vicari et al., 1996, 2003). Thus, a comparison group may be matched on verbal (e.g., Peabody Picture Vocabulary Scale) or nonverbal (e.g., Corsi Blocks) with the consequent impact on the increasing or decreasing of control group chronological age. The non-uniformity in the criteria used in selecting comparison groups makes data interpretation difficult with respect to different memory systems that have different developmental trajectories. A possible alternative, used in this study, is to use the chronological age as the matching criteria in order to have a clearer picture of the memory functioning of WS when taking normal development as comparison population.

Indeed, results from our study suggest that memory impairment in WS is developmental delayed and is associated with intellectual commitment and visuo-processing problems, rather than a dissociation within different memory systems. These results are inconsistent with data from previous studies (Nichols et al., 2004; Vicari et al., 1996) and deserves further discussion.

A dissociation hypothesis between short- and long-term storage has been proposed to characterize memory abnormalities observed in WS (Nichols et al., 2004; Vicari et al., 1996). Taking into account that WS subjects did not exhibit primacy effect, Vicari et al. (1996) interpreted their results as an evidence for dissociation in the two memory storages. The primacy and recency effect would reflect the content of different memory storage models (a long- and short-term storage system) suggesting that a deficit in long-term memory coexisted with preservation of shortterm memory. This hypothesis was also advanced by Nichols et al. (2004). However, in our study, in spite of a reduced span in each serial position, a normal serial position curve was observable in both groups. Our data suggest that, in face of a decrease in immediate memory span, WS 
and control groups show similar patterns in information coding, storage and recall. Indeed, consistent with previous studies (Brock et al., 2006), WS performance in verbal learning test show that, although they exhibit lower immediate memory span, they are able to learn and retain new information. Moreover, it is important to note that the loss of information after a retention interval is a natural process that occurred in normal development individuals as well. Decreases in WS immediate verbal memory span may be related not only with the inability to use effectively semantic or serial coding strategies, but also with high interference susceptibility (intrusions and false positives) and poor item discrimination, previously reported (Nichols et al., 2004; Vicari et al., 1996). This type of difficulties clearly suggests that overall intellectual functioning is associated with WS memory impairments. Thus, several authors have found evidence for a strong association between working memory and intelligence (Colom, Flores-Mendoza, Quiroga, \& Privado, 2005; Colom, Rebollo, Palacios, Juan-Espinosa, \& Kyllonen, 2004; Verguts \& Boeck, 2002). Also, the results of the present study bring additional confirmation for understanding poor performance in Corsi Blocks of WS subjects, which is also observed in Digit Span. This combined deficit in Corsi Blocks and Digit Span tests seems to be inconsistent with the dissociation hypothesis (Jarrold et al., 1999). Again, although WS subjects showed inferior results, we found similar patterns of performance in WS and control group. First, no differences between groups were found when verbal span (Digit Span) performance in forward and backward conditions was compared, with equal patterns of lower performance in the backward condition in both groups. These data are consistent with other studies in normal development (Mammarella \& Cornoldi, 2005). Importantly, mean span for this clinical group, obtained in forward and backward order is consistent with other studies (Mervis et al., 1999; Wang \& Bellugi, 1994). Second, other interesting result has to do with the presence of the same decreasing pattern for visuo-spatial items with respect to IMS for digits in both WS and control groups. This evidence is consistent with developmental trajectories for normal development, showing an advantage of 1.5 items of the verbal span over spatial span (Nichelli, Bulgheroni, \& Riva, 2001), which was also found in both groups in this study. In addition, difficulties in processing visuo-spatial stimuli may also be underlying this poor performance in Corsi Blocks test. Third, when relative levels of impairments were analyzed in WS group ( $z$ scores in verbal and visuo-spatial short- and long-term memory tests), no significant differences were found within performance across different memory tests. Finally, when age subgroups were analyzed, the same patterns were observed in both groups, suggesting that changes in performance across development are similar in WS and normal controls.

Results in Rey-Osterrieth Complex Figure suggest that WS poor performance may be related with their visuo-constructive difficulties, necessary to accomplish the copy con- dition. In fact, although they did not differ in the figure approaching strategy, they reproduced and recalled significantly lower structural and incidental details. However, when compared the number of reproduced elements in copy and recall conditions, the loss of visual information occurred similarly in both groups. These data suggest that visuo-processing deficits, a core characteristic of WS cognitive profile (Mervis et al., 2000), might be related with their visuo-spatial long-term memory deficits. Indeed, recent studies proposed that visuo-spatial long-term memory deficits observed in WS may be secondary to their difficulties in visuo-spatial processing (Jarrold et al., 2007).

The current study suggests that memory performance in our WS group may reflect developmental delays rather than deviant dissociative patterns. Also, overall intellectual functioning and visuo-spatial deficits, a main feature of WS cognitive profile (Mervis et al., 2000), may be associated with difficulties in WS performance on memory tasks. As working memory abilities are closely related with learning abilities, structured cognitive interventions that address these patterns of memory performance are important in the cognitive rehabilitation of these patients. In fact, there is evidence suggesting that WS patients are able to engage in rehearsal training (Brock et al., 2006) and benefit from cognitive training (Gonçalves et al., 2005). Thus, training memory abilities, either short-term or long-term, with simple instructions, may contribute to the improvement of WS patients memory performance and ultimately have an impact on their learning abilities (Gathercole \& Alloway, 2006). Future studies need to be done in order to replicate these current findings with a larger sample size and the use of multiple comparisons groups including verbal, nonverbal and subject to memory training programs.

\section{Acknowledgments}

This research was supported by the Grants POCTI/PSI/ 58364/2004 and SFRH/BD/16091/2004 from Fundação para a Ciência e Tecnologia (Portugal).

\section{References}

Baddeley, A. D., \& Hitch, G. (1974). Working memory. In G. H. Bower (Ed.). The psychology of learning and motivation: Advances in research and theory (Vol. 8, pp. 47-89). New York: Academic Press.

Bellugi, U., Lichtenberger, L., Jones, W., Lai, Z., \& St. George, M. (2000). I. The neurocognitive profile of Williams syndrome: A complex pattern of strengths and weaknesses. Journal of Cognitive Neuroscience, 12(Suppl. 1), 7-29.

Bellugi, U., Wang, P. P., \& Jernigan, T. J. (1994). Williams syndrome: An unusual neuropsychological profile. In S. B. J. Grafman (Ed.), Atypical cognitive deficits in developmental disorders: Implications for brain function. Hillsdale, NJ: Lawrence Erlbaum Associates.

Bernstein, J. H., \& Waber, D. P. (1996). Developmental scoring system for the Rey-Osterreith Complex Figure: Professional manual. Odessa, FL: Psychological Assessment Resources, Inc..

Brock, J., Brown, G. D., \& Boucher, J. (2006). Free recall in Williams syndrome: Is there a dissociation between short- and long-term memory? Cortex, 42(3), 366-375. 
Colom, R., Flores-Mendoza, C., Quiroga, M. A., \& Privado, J. (2005). Working memory and general intelligence: The role of short-term storage. Personality and Individual Differences, 39, 1005-1014.

Colom, R., Rebollo, I., Palacios, A., Juan-Espinosa, M., \& Kyllonen, P. (2004). Working memory is (almost) perfectly predicted by $\mathrm{g}$. Intelligence, 32, 277-296.

Corsi, P. M. (1972). Human memory and the medial temporal region of the brain. Unpublished doctoral thesis, McGill University, Montreal, Canada.

Delis, D. C., Kramer, J. H., Kaplan, E., \& Ober, B. A. (1994). California Verbal Learning Test-children's version: Manual. San Antonio: The Psychological Corporation.

Delis, D. C., Kramer, J. H., Kaplan, E., \& Ober, B. A. (2000). California Verbal Learning Test-Second Edition, adult version, manual. San Antonio: The Psychological Corporation.

Farran, E. K., \& Jarrold, C. (2005). Evidence for unusual spatial location coding in Williams syndrome: An explanation for the local bias in visuo-spatial construction tasks? Brain and Cognition, 59(2), 159-172.

Gagliardi, C., Martelli, S., Burt, M. D., \& Borgatti, R. (2007). Evolution of neurologic features in Williams syndrome. Pediatric Neurology, 36(5), 301-306.

Gathercole, S. E., \& Alloway, T. P. (2006). Practitioner review: Short-term and working memory impairments in neurodevelopmental disorders: Diagnosis and remedial support. Journal of Child Psychology and Psychiatry, and Allied Disciplines, 47(1), 4-15.

Gonçalves, O. F., Pérez, A., Henriques, M., Prieto, M., Lima, M., Siebert, M., et al. (2004). Funcionamento Cognitivo e Produção Narrativa no Síndrome de Williams: Congruência ou Dissociação Neurocognitiva? International Journal of Clinical and Health Psychology, 4, 623-638.

Gonçalves, O. F., Prieto, M., Sampaio, A., Pérez, A., Henriques, M., ReisLima, M., et al. (2005). Cognitive profile in Williams syndrome: A case study. British Journal of Developmental Disabilities, 51, 149-159.

Hoffman, J. E., Landau, B., \& Pagani, B. (2003). Spatial breakdown in spatial construction: Evidence from eye fixations in children with Williams syndrome. Cognitive Psychology, 46(3), 260-301.

Jarrold, C., Baddeley, A. D., \& Hewes, A. K. (1999). Genetically dissociated components of working memory: Evidence from Down's and Williams syndrome. Neuropsychologia, 37(6), 637-651.

Jarrold, C., Baddeley, A. D., \& Phillips, C. (2007). Long-term memory for verbal and visual information in Down syndrome and Williams syndrome: Performance on the Doors and People test. Cortex, 43(2), 233-247.

Korenberg, J. R., Chen, X. N., Hirota, H., Lai, Z., Bellugi, U., Burian, D., et al. (2000). VI. Genome structure and cognitive map of Williams syndrome. Journal of Cognitive Neuroscience, 12(Suppl. 1), 89-107.
Mammarella, I. C., \& Cornoldi, C. (2005). Sequence and space: The critical role of a backward spatial span in the working memory deficit of visuospatial learning disabled children. Cognitive Neuropsychology, 26, 1055-1068.

Mervis, C. B., Morris, C. A., Bertrand, J., \& Robinson, B. F. (1999). Williams syndrome: Findings from an integrated program of research. In H. Tager-flsuberg (Ed.), Neurodevelopmental disorders (pp. 65-110). Boston: MIT Press.

Mervis, C. B., Robinson, B. F., Bertrand, J., Morris, C. A., KleinTasman, B. P., \& Armstrong, S. C. (2000). The Williams syndrome cognitive profile. Brain and Cognition, 44(3), 604-628.

Metcalfe, K. (1999). Williams syndrome: An update on clinical and molecular aspects. Archives of Disease in Childhood, 81(3), 198-200.

Nichelli, F., Bulgheroni, D., \& Riva, D. (2001). Developmental patterns of verbal and visuospatial spans. Neurological Sciences, 22, 377-384.

Nichols, S., Jones, W., Roman, M. J., Wulfeck, B., Delis, D. C., Reilly, J., et al. (2004). Mechanisms of verbal memory impairment in four neurodevelopmental disorders. Brain and Language, 88(2), $180-189$.

Rey, A. (1959). Manuel du test de copie d'une figure complexe de A. Rey. Paris: Les Editions du Centre de Psychologie Appliquée.

Stromme, P., Bjornstad, P. G., \& Ramstad, K. (2002). Prevalence estimation of Williams syndrome. Journal of Child Neurology, 17(4), 269-271.

Verguts, T., \& Boeck, P. (2002). On the correlation between working memory capacity and performance on intelligence tests. Learning and Individual Differences, 13, 37-55.

Vicari, S., Bellucci, S., \& Carlesimo, G. A. (2003). Visual and spatial working memory dissociation: Evidence from Williams syndrome. Developmental Medicine and Child Neurology, 45(4), 269-273.

Vicari, S., Bellucci, S., \& Carlesimo, G. A. (2005). Visual and spatial longterm memory: Differential pattern of impairments in Williams and Down syndromes. Developmental Medicine and Child Neurology, 47(5), 305-311.

Vicari, S., Brizzolara, D., Carlesimo, G. A., Pezzini, G., \& Volterra, V. (1996). Memory abilities in children with Williams syndrome. Cortex, 32(3), 503-514.

Wang, P. P., \& Bellugi, U. (1994). Evidence from two genetic syndromes for a dissociation between verbal and visual-spatial short-term memory. Journal of Clinical and Experimental Neuropsychology, 16, 317-322.

Wechsler, D. (1991). Wechsler Intelligence Scale for children. Manual (3rd ed.). San Antonio: Psychological Corporation.

Wechsler, D. (1997). Wechsler Adult Intelligence Scale. Manual (3rd ed.). San Antonio: Psychological Corporation. 